\title{
Ciclo de presentación de libros: reseñas geográficas. Debates, perspectivas y reflexiones de la geografía local en el contexto global
}

\author{
LETICIA MANASSERO \\ Universidad Nacional del Litoral ${ }^{1}$ \\ letmanassero@hotmail.com
}

Durante los meses de octubre y noviembre del año 2020, se llevó a cabo el Ciclo de presentaciones de libros "Reseñas Geográficas. Debates, perspectivas y reflexiones de la geografia local en un contexto global", organizado por el Departamento de Geografía de la Facultad de Humanidades y Ciencias (FHUC) de la Universidad Nacional del Litoral (UNL) y por Revista Locale, Revista Universitaria de Geografía (FHUC-UNL). Se trató de un evento que contó con cuatro encuentros virtuales, en los cuales participaron exponentes docentes, investigadores, graduados, comentaristas y participantes en general. El ciclo tuvo como objetivo socializar las producciones recientes de los integrantes del Departamento de Geografía (FHUC-UNL), a partir de la presentación de cuatro libros que abordan diversas líneas de trabajo, debates, problemáticas y discusiones en torno al quehacer geográfico.

En el primer encuentro, que tuvo lugar el 8 de octubre, se presentó el libro: "Geografía de Santa Fe. Transformaciones recientes del territorio provincial” (Oscar Lossio y Gustavo Peretti, 2020). Los compiladores del libro realizaron una presentación de la obra colectiva e hicieron referencia a la importancia de compilar y socializar las producciones realizadas por docentes e investigadores del Departamento de Geografía, elemento que rescató María Luisa D’Angelo, ex docente de la casa de estudios y autora del prólogo del libro. La geógrafa se refirió a la importancia de visibilizar las producciones geográficas en nuestra región, no solo por su impronta en la formación de estudiantes y docentes de

1 Licenciada en Geografía egresada de la Facultad de Humanidades y Ciencias, UNL. 
enseñanza media y superior, sino también por su potencial de generar instrumentos para la toma de decisiones políticas. En tal sentido, manifestó la importancia de la divulgación científica y de la socialización de las propuestas de trabajo que llevan a cabo los/las geógrafos/as en múltiples medios.

Los autores de los artículos que componen el libro dieron continuidad al encuentro presentando sus respectivos trabajos. Oscar Lossio expuso el contenido del Capítulo I, en el cual abordó las transformaciones de los espacios rurales en el distrito Matilde desde 1990, a partir de una perspectiva teórico-metodológica constructivista que recupera la mirada y las experiencias de los productores. Mariela Demarchi expuso el Capítulo 2, referido a la dimensión espacial de la migración boliviana hacia el cinturón hortícola santafesino desde 1970, a partir de un estudio centrado en la teoría de las redes sociales en dichos procesos migratorios. María Mercedes Cardoso se refirió al Capítulo 3, en el cual realiza un desarrollo teórico del concepto de contraurbanización y analiza sus implicancias en el caso del Área metropolitana de Santa Fe. Javier Gómez y Gustavo Peretti presentaron el Capítulo 4, en el que abordan los cambios y diferencias regionales en la distribución poblacional y el sistema de asentamientos de la provincia de Santa Fe en el período I895-2010. Carina Davies expuso el Capítulo 5, en el cual explora estrategias de desarrollo territorial en ciudades intermedias, centrando su análisis en Reconquista y Venado Tuerto. Finalmente, Norma Finelli y María Mercedes Cardoso presentaron el Capítulo 6, referido a las transformaciones en el puerto de la ciudad de Santa Fe a partir de inversiones privadas asociadas a procesos de reconversión y rehabilitación urbana, que tuvieron lugar a partir de la década de 1990.

El día 29 de octubre se llevó a cabo el segundo encuentro del ciclo, en el cual se presentó el libro: "La educación agrotécnica en la Universidad Nacional del Litoral” (Salomón y Leonardi, 2018). El encuentro contó con la participación como exponente de Roberto Leonardi, uno de los autores del libro y docente de la Facultad de Humanidades y Ciencias y de la Facultad de Ciencias Agrarias (UNL). Durante su intervención recuperó parte del contenido del libro, que conjuga un análisis con perspectiva histórica, junto con voces de actores y testimonios en torno a la Escuela de Agricultura, Ganadería y Granja, fundada en la década de 1960 en Esperanza (Santa Fe, Argentina) y dependiente de la UNL. El 
libro incorpora un análisis centrado en el territorio y los actores sociales, en especial los actores agrarios de la región. A su vez, pretende aportar a la historia institucional de la escuela y a la recuperación de los sentidos de pertenecía de quienes la construyen día a día.

La presentación dio lugar a debates en los que el autor tuvo la oportunidad de explayarse en relación con las dificultades que atraviesa la institución actualmente. En parte, estas dificultades radican en la disminución de la importancia del farmer (colono) como actor social clave. A diferencia de otras escuelas agrotécnicas, la Escuela de Agricultura, Ganadería y Granja de Esperanza -conocida como Escuela Granja- se consolida luego del pacto territorial farmer (1856-1929/30), nace a fines de un pacto territorial cooperativo (I940-1970), por lo que irá ganando importancia con el pasar del tiempo la formación de futuros asalariados. A este proceso se suman las consecuencias de las lógicas impuestas por el pacto territorial de comodities (1980/...), tanto en Esperanza como de otras áreas rurales argentinas. En el avance de la economía centrada en los comodities interesan otros cultivos y otras lógicas de producción, que se territorializan desplazando actores sociales, borrando procesos identitarios y modificando el paisaje.

Durante el tercer encuentro, llevado a cabo el 5 de noviembre, se presentó el libro: "Las condiciones de riesgo ambiental en los pueblos de la costa" (Visintini, en prensa). María Laura Bertuzzi realizó una presentación del libro, destacando su relevancia en el contexto actual, la trama y los hilos conductores de los cinco capítulos y los debates y discusiones que convergen en las conclusiones. Posteriormente, María Laura Visintini, coordinadora del libro, narró la historia de la publicación y las trayectorias previas de investigación y extensión que tuvieron lugar desde el año 2006 y que permitieron la escritura de la obra colectiva. Seguidamente, los autores expusieron en orden los cinco capítulos de la obra.

María Laura Visintini hizo un recorrido sobre el Capítulo I, escrito por Hortensia Castro. En este se presentan herramientas analíticas en torno al concepto multidimensional de vulnerabilidad y a la construcción social del riesgo. Los conceptos allí desarrollados son centrales en el resto del libro y aportan a la búsqueda de las causas estructurales que configuran el riesgo ambiental en la zona de la costa. Juan Pablo Levrino y Manuel del Rey Rodríguez expusieron el Capítulo 2, en su trabajo abordan los rasgos físicos del territorio de la costa, en el 
marco de una perspectiva que concibe dichas características físicas y la sociedad de forma relacional. María Laura Visintini presentó el Capítulo 3, que se enfoca en los componentes sociodemográficos de la vulnerabilidad, a partir del análisis de censos de población y considerando múltiples variables en relación, que contribuyen al objeto de análisis. Carolina Schmidt presentó el Capítulo 4, la autora apunta, desde un enfoque centrado en las cadenas productivas, al estudio de las vulnerabilidades económico-productivas, adentrándose en las diversas actividades económicas, los usos productivos y su relación con el riesgo ambiental del área. Finalmente, Pilar Cabré y Ma. Laura Visintini presentaron el Capítulo 5, las autoras expusieron sobre las vulnerabilidades desde un análisis político e institucional, conjugando las acciones de diversos actores sociales.

El último de los encuentros se realizó el I2 de noviembre, ocasión en la cual se presentó el libro "Desafios de la Geografía. Teorias, métodos y perspectivas" (Alfaro; Cardozo; Davies; Seval; Arnaudo, 2020). En este caso, el evento contó con la participación de Timo Bartthol (UFF) y Juan Manuel Diez Tetamanti (CONICET) como comentaristas. Los textos que comprende la obra tienen como autores a María Luisa D’Angelo, Blanca Ramírez, Joaquín Bosque Sendra, Josefina Gómez Mendoza, Hortensia Castro, Jorge Montenegro, Perla Zusman y Alicia Lindón.

Para comenzar, Carina Davies realizó una breve reseńa sobre la historia del libro, que fue publicado originalmente en el año 2014 en formato papel, y editado nuevamente en formato digital y de libre acceso en el año 2020. Se trató de un proyecto editorial autogestivo, que surgió en el marco del grupo "Geográficos", formado por estudiantes del profesorado y licenciatura de la facultad. La obra fue realizada en base a videoconferencias organizadas por el grupo en el año 2007 y el año 2009 con un posterior trabajo de compilación y de edición por parte de los autores. El propósito del libro fue contribuir a los debates sobre la ciencia geográfica -concebida en diálogo con otras disciplinas-, problematizando su objeto de estudio y las perspectivas teórico-metodológicas.

Posteriormente, los comentaristas y otros compiladores realizaron un recorrido por el contenido de algunos capítulos, haciendo mención a la riqueza del libro en lo que respecta a la incorporación de perspectivas críticas, latinoamericanas, abordajes desde la periferia y giros disciplinares. Asimismo, se destacó la particular innovación que supuso la realización 
de videoconferencias tempranamente, haciendo un paralelismo con el contexto actual. Finalmente, hubo otras intervenciones que articularon los contenidos del libro con temas vigentes, junto con apreciaciones en torno al rol y los desafíos de los/las geógrafos/as en la actualidad.

En los cuatro encuentros se dieron interesantes debates entre el público asistente y quienes expusieron el contenido de los libros presentados. Entre los tópicos de los debates se destacan: el rol de la geografía, el lugar que ocupa en la toma de decisiones, el vínculo entre los debates teórico y la sociedad, el vínculo entre la universidad y la escuela media, entre otros debates relacionados a transformaciones territoriales vinculadas al campo específico de cada presentación. En síntesis, luego de realizar un recorrido por los encuentros que formaron parte del evento ${ }^{2}$, podemos decir que el ciclo de presentaciones virtuales fue un espacio de diálogo y socialización que habilitó la continuidad del intercambio en el contexto de pandemia. Asimismo, a partir de los cuatro encuentros, pudo apreciarse la diversidad de aportes y perspectivas en las producciones de los y las integrantes del Departamento de Geografía (FHUC-UNL). Las obras presentadas algunas en formato digital y de libre acceso- son aportes relevantes para la formación de docentes e invitan a dar continuidad a líneas de trabajo y, al mismo tiempo, habilitan nuevas discusiones.

2 Agradecemos la gentileza de Gustavo Aressi Cataccio, por colaborar con sus anotaciones para la elaboración de esta reseña 


\section{Bibliografía}

\section{Alfaro, M.B.; Cardozo, L.; Davies, C.; Seval, M. y Arnaudo, J.P. (Comps.)}

(2020). Desafios de la Geografía. Teorías, métodos y perspectivas, Santa Fe:

Ediciones UNL. [En línea] Recuperado de: https://bibliotecavirtual.unl.edu. ar:8443/handle/III $85 / 5580$

Lossio, O. y Peretti, G. (comps.) (2020). Geografía de Santa Fe.

Transformaciones recientes del territorio provincial, Santa Fe: Ediciones

UNL. [En línea] Recuperado de: https://bibliotecavirtual.unl.edu.ar:8443/ handle/1II85/5572

Salomón, P.; Leonardi, R. et al. (2018). La educación agrotécnica en la Universidad Nacional del Litoral, Santa Fe: Ediciones UNL

Visintini, M. (Coord.) (en prensa). Las condiciones de riesgo ambiental en los pueblos de la costa, Santa Fe: Ediciones UNL. 